\title{
Progression of Barrett's esophagus toward esophageal adenocarcinoma: an overview
}

\author{
Nele Schoofs, Raf Bisschops, Hans Prenen
}

University Hospitals Leuven, Leuven, Belgium

\begin{abstract}
In Barrett's esophagus, normal squamous epithelium is replaced by a metaplastic columnar epithelium as a consequence of chronic gastroesophageal reflux disease. There is a strong association with esophageal adenocarcinoma. In view of the increasing incidence of esophageal adenocarcinoma in the western world, it is important that more attention be paid to the progression of Barrett's esophagus toward esophageal adenocarcinoma. Recently, several molecular factors have been identified that contribute to the sequence towards adenocarcinoma. This might help identify patients at risk and detect new targets for the prevention and treatment of esophageal adenocarcinoma in the future.
\end{abstract}

Keywords Barrett's esophagus, esophageal adenocarcinoma, biomarkers

Ann Gastroenterol 2017; 30 (1): 1-6

\section{Introduction}

In view of the increasing incidence of esophageal adenocarcinoma (EAC) in the Western world, it is important to better understand the process of neoplastic progression of Barrett's esophagus (BE) toward EAC. In this review we will focus on the known risk factors for this progression, as well as the molecular pathways involved. We searched PubMed for articles published in English from 2000 onwards and used the search terms "esophageal cancer", "Barrett's esophagus", "etiology", "pathology”, "molecular pathogenesis", "genetics", "pathophysiology", "diagnosis", “epidemiology”, and "chemoprevention".

\section{Definition of BE}

$\mathrm{BE}$ is most commonly seen as the condition in which a metaplastic columnar epithelium replaces the stratified squamous epithelium that normally lines the distal esophagus $[1,2]$. The metaplastic epithelium is acquired as

Department of Gastroenterology, University Hospitals Leuven and Department of Oncology, KU Leuven, Belgium

Conflict of Interest: None

Correspondence to: Hans Prenen, MD, PhD, University Hospitals Leuven, Department of Gastroenterology, Digestive Oncology Unit, Herestraat 49, B3000 Leuven, Belgium, Tel.: +32 163442 18, Fax: +32 163444 19, e-mail: hans.prenen@uzleuven.be

Received 10 June 2016; accepted 12 September 2016; published online 30 September 2016

DOI: http://dx.doi.org/10.20524/aog.2016.0091 a consequence of chronic gastroesophageal reflux disease (GERD), and is a predisposing factor for the development of adenocarcinoma of the esophagus.

There are many theories concerning the origin of a $\mathrm{BE}$ and no consensus has been reached, as was stated by the authors of a recent review [3]. They concluded that Barrett glands are more complex and possibly unique within the human gastrointestinal epithelium. Barrett epithelium shows multilinear gastric and intestinal differentiation, as documented by the findings of gene expression arrays of both gastric and intestinal epithelium and the different types (complete versus incomplete) of intestinal metaplasia. This could lead to different hypotheses concerning the development of $\mathrm{BE}$ and the possible progression to EAC.

\section{Epidemiology}

It is difficult to determine the epidemiology of BE because there are many affected individuals who are asymptomatic and remain undiagnosed. Most prevalence data have been derived from $\mathrm{BE}$ diagnoses made during esophagogastroduodenoscopy performed to investigate symptoms of dyspepsia. BE is more common in developed countries, affecting $2 \%$ of the general adult population [4]. This is most likely to be attributable to the higher incidence of GERD in this population, since this is a well-known causal factor for the development of BE.

The incidence of $\mathrm{BE}$ on diagnostic endoscopy is rising independently of an increase in the number of endoscopies carried out, suggesting a true increase in incidence rather than a higher detection rate [5]. In the literature, several publications have studied the prevalence of $\mathrm{BE}$ in unselected populations. Rex et al evaluated a cohort of 961 patients undergoing colonoscopy who were offered an additional endoscopy, and found an overall prevalence of $6.8 \%$, with $5.5 \%$ 
for short-segment BE in persons aged 40 years or older [6]. In another similar colonoscopy-based study of 300 patients over the age of 65 years, the prevalence was $4 \%$ and $15 \%$ for long- and short-segment BE, respectively [7]. In other population-based studies, the prevalence of $\mathrm{BE}$ in the general population ranged between $1.3 \%, 1.6 \%$ and $1.9 \%$ [8-10].

The incidence of esophageal cancer is highly variable, depending on the region, with "'the esophageal cancer belt" as the highest-risk area for the development of esophageal cancer, stretching from northern Iran through the central Asian republics to north-central China. In this area however, $90 \%$ of cases are squamous cell carcinomas. Over the past decades, the frequency of adenocarcinoma of the esophagus has increased dramatically, certainly in western countries ("low-risk areas"). Several hypotheses have been proposed concerning the increasing incidence of $\mathrm{BE}$ and consequently of EAC. The first hypothesis is that the lower prevalence of Helicobacter pylori (H. pylori) infection, particularly the cagA + strain, is associated inversely with $\mathrm{BE}$ [11]. A second hypothesis is the increase in the known risk factors of overweight and obesity $[12,13]$.

\section{Diagnosis}

A diagnosis of $\mathrm{BE}$ is made endoscopically by visualization of a columnar lined epithelium arising circumferentially at least $1 \mathrm{~cm}$ above the gastroesophageal junction, with intestinal metaplasia on histological investigation of biopsies. Endoscopic reporting can be difficult and should be done using uniform criteria. The Prague classification consists of criteria to uniformly describe the length of a Barrett's segment during endoscopy. In this classification, the circumferential extent and maximum length of the Barrett epithelium are described in $\mathrm{cm}$ (Fig. 1). This classification was recently validated [14].

For a definitive diagnosis of $\mathrm{BE}$, histological diagnosis is necessary. The presence of specialized columnar epithelium, characterized by acid mucin-containing goblet cells, in a biopsy specimen of the esophagus has been accepted as diagnostic of $\mathrm{BE}$ [15]. Endoscopic surveillance of BE with systematic biopsies is necessary to exclude the presence of dysplasia.

$\mathrm{BE}$ can be divided into short- and long-segment $\mathrm{BE}$. Short-segment $\mathrm{BE}$ has a maximal length of less than $3 \mathrm{~cm}$, whereas long-segment has a length of more than $3 \mathrm{~cm}$. Long-segment BE has a higher risk for development of EAC. The length of the BE segment is known to be associated with risk of progression to neoplasia, as discussed below.

\section{Risk for progression to EAC}

Incidence rates of EAC and high-grade dysplasia (HGD) among patients with $\mathrm{BE}$ are variable. Seven systematic reviews have been published on the cancer risk in patients with $\mathrm{BE}$. The annual incidence of EAC among BE patients varied from $0.3 \%$ to $0.6 \%$, and the combined incidence of HGD and EAC from $0.9 \%$ to $1.0 \%$ [16-22]. These incidence rates are still referred to in recent guidelines. One study, however, revealed a publication bias in the reporting of progression rates in

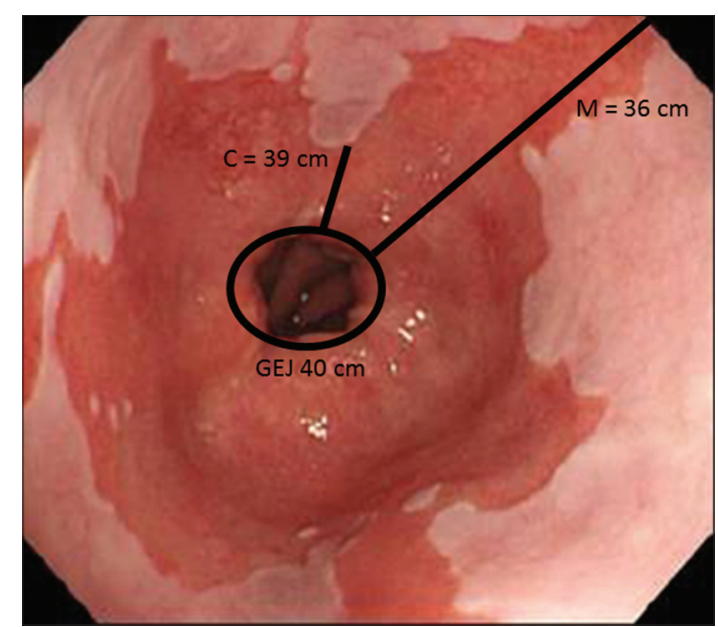

Figure 1 The Prague classification of Barrett's esophagus. The C-value is used for the circumferential pattern (C) and the $\mathrm{M}$ value for the maximum length $(\mathrm{M})$

GEJ, gastroesophageal junction

patients with BE [16]. Sikkema et al analyzed data derived from high-quality studies to obtain more accurate data on the risk of EAC in BE patients [20]. The annual EAC incidence rate in BE cohorts with less than 2000 patient years of follow up was $0-3.55 \%$, compared to $0.07-0.82 \%$ in cohorts with more than 2000 patient years [20]. In two population-based BE follow-up studies, absolute annual risks of $0.12-0.14 \%$ were reported, considerably lower than was assumed until now [23,24].

Considering the known risk for the progression of $\mathrm{BE}$ toward EAC, current guidelines advise patients with $\mathrm{BE}$ to be enrolled in endoscopic surveillance programs in order to detect HGD or EAC. Endoscopic surveillance consists of extensive biopsy sampling, known as the Seattle biopsy protocol. This protocol consists of four quadrant biopsies (every 1-2 cm) with biopsies of mucosal abnormalities. The surveillance interval is determined by histology results. In the absence of dysplasia, the American College of Gastroenterology recommends surveillance endoscopy at 3-year intervals. For patients with low-grade dysplasia, an annual endoscopy is recommended, and for those with HGD who receive no invasive therapy, endoscopic exams should be performed every three months. In a recent review [12], the authors discuss the current guidelines and the dilemmas in endoscopic surveillance. Several studies suggest a modest effect of surveillance programs on EAC mortality in patients with BE [25-27]. This outcome is probably influenced by the estimates of annual incidence of EAC among patients with $\mathrm{BE}$, considering the fact that the incidence employed in current surveillance guidelines is still $0.4-0.5 \%$, which is much lower than discussed above. Another dilemma in surveillance strategies for $\mathrm{BE}$ is the possibility of sampling error and poor adherence to the Seattle protocol, which has been reported to be as low as $30 \%$ [28]. To improve the success of endoscopic surveillance, advanced endoscopic techniques, such as chromoendoscopy, narrow-band imaging (NBI) and autofluorescence endoscopy, have attracted major interest during the past decade. In 2012, a prospective, randomized, controlled trial compared the 
standard endoscopic surveillance strategy with NBI-targeted biopsies, with similar results in diagnosing intestinal metaplasia and even detecting more areas with dysplasia [29]. When using NBI-targeted strategies, fewer biopsies are necessary, which could increase cost-effectiveness. Some authors conclude that this technique should, therefore, be considered in patients with longer segments of $\mathrm{BE}$, considering the poorer adherence in these cases [28]. This is not supported in recent British Society of Gastroenterology (BSG) guidelines, considering the conflicting results in the literature and the lack of hard evidence. According to the BSG guidelines, however, advanced imaging modalities such as chromoendoscopy or "virtual chromoendoscopy" are not superior to standard white light endoscopy in BE surveillance and are therefore not recommended for routine use [30]. There is also a bias concerning the incidence rate of EAC in these studies, as stated previously.

The grading of dysplasia in these guidelines is also a concern in view of the poor inter- and intra-observer histological agreement as to the diagnosis of Barrett's dysplasia [31], as an inaccurate grading of dysplasia can impact the frequency of endoscopic surveillance.

Ross-Innes et al (BEST2 Study Group) described the use of Cytosponge as a minimally invasive technique for screening of BE [32]. They used Trefoil factor 3 (TFF3) as a marker of intestinal metaplasia in the Cytosponge samples, as it is the subtype most strongly associated with a risk of progression. In a study with 463 controls and $467 \mathrm{BE}$ patients, the Cytosponge test appeared to be a safe and well-tolerated BE screening method that can be carried out in a primary care setting. The prevalence of $\mathrm{BE}$ of $3.0 \%$ reported in this study is comparable with other studies. The sensitivity of the combination test (Cytosponge + TFF3) is approximately $80 \%$ for diagnosing BE; this increases with BE segment length and is not compromised in the presence of dysplasia. The specificity of the test was $92 \%$. In a microsimulation model, both endoscopy and Cytosponge were compared, with no screening, in 50-yearold men with symptoms of GERD [33]. This already showed cost-effectiveness and reduced mortality from EAC for the Cytosponge test compared with no screening.

\section{Molecular pathways}

The progression from BE to EAC was first documented in the 1970's, providing targets for the screening, monitoring, and management of early-stage neoplasia [34]. Currently, there are several risk factors described as possible predictors of progression. The Prasad's group published a review in 2010, summarizing the current evidence on risk factors for progression [35]. An attempt was made to create a potential risk stratification to optimize the management of patients with $\mathrm{BE}$ and make it more cost-effective. A progression score for $\mathrm{BE}$ could contain a group of clinical factors and a biomarker panel. In the recent literature, there are certain factors that are suggested as being important risk factors. The clinical panel consists of age, (male) sex, and length of Barrett segment, with increasing length giving an increasing risk but without an evident cut off length. Biomarkers included are aneuploidy/ polysomy, p53 loss of heterozygosity (LOH) and p16 LOH. This possible score, however, certainly needs validation in a prospective study of a large cohort of patients before it can be used in clinical practice. If validated, this score could be important for determining which patients are at higher risk of developing an adenocarcinoma and would therefore benefit the most from inclusion in a program of intensive surveillance. A familial aggregation of BE has also been described [36].

Obesity is implicated as a risk factor for EAC and BE, independently of GERD [37]. Thrift et al described a possible role for high levels of circulating pro-inflammatory cytokines and leptin in the mechanism towards progression [38]. In another analysis, an inverse association between high molecular weight adiponectin levels and risk of progression to EAC is reported [39].

A possible sequence for progression is described in a hypothesis by Chandrasoma et al [40], derived from the idea of BE as a complex, multilinear epithelium with different types of metaplasia (cf. definition). The authors suggest an evolution from the esophageal squamous epithelium to cardiac-type glands and further into intestinal metaplastic glands, which can progress to neoplasia. A concept of Barrett glands evolving from metaplasia of the stem cells of the proximal columnar gastric or cardiac epithelium has been stated [41].

In the recent literature, much attention has been given to understanding the molecular pathways leading to the progression from BE to adenocarcinoma of the esophagus, in order to specify possible therapeutic targets in the prevention and treatment of this type of (early) cancer (Fig. 2). Several embryological signaling pathways are described in the development and malignant transformation of $\mathrm{BE}$ [42]. In embryology, the esophagus is derived from the foregut, whose lumen divides along the sagittal axis into the trachea, with columnar epithelium, and the esophagus, with squamous epithelium. Studies of transgenic mouse models have identified four main signaling pathways active in the differentiation of the embryological foregut: the bone morphogenetic protein (BMP), hedgehog ( $\mathrm{Hh}$ ), winglesstype MMTV integration site family (WNT), and retinoic acid (RA) signaling pathways [42]. The three key transcription factors expressed by these pathways for the regulation of differentiation of foregut epithelium toward a squamous or columnar type are NKX2.1, SOX2 and p63. SOX2 and p63 induce squamous differentiation, while NKX2.1 expression is required for columnar differentiation of the foregut epithelium. These pathways and transcription factors not only play an important role in foregut embryology, but may also be important in the development of BE and its progression toward EAC. In normal squamous epithelium, the BMP pathway is not activated, but in the case of inflammation (such as that caused by GERD) the BMP pathway is activated with stromal BMP4 expression, which contributes to a columnar transdifferentiation of squamous esophageal epithelium. The role of this BMP pathway in the progression toward adenocarcinoma requires further research. The Hh pathway is also involved in the development of BE by stimulating the BMP pathway, but it can also act by inducing a transcription factor, epithelial SOX9 expression. Its role in malignant progression is less clear. In contrast, WNT signaling is not involved in the development of $\mathrm{BE}$, but is an important factor in its progression toward EAC. This 


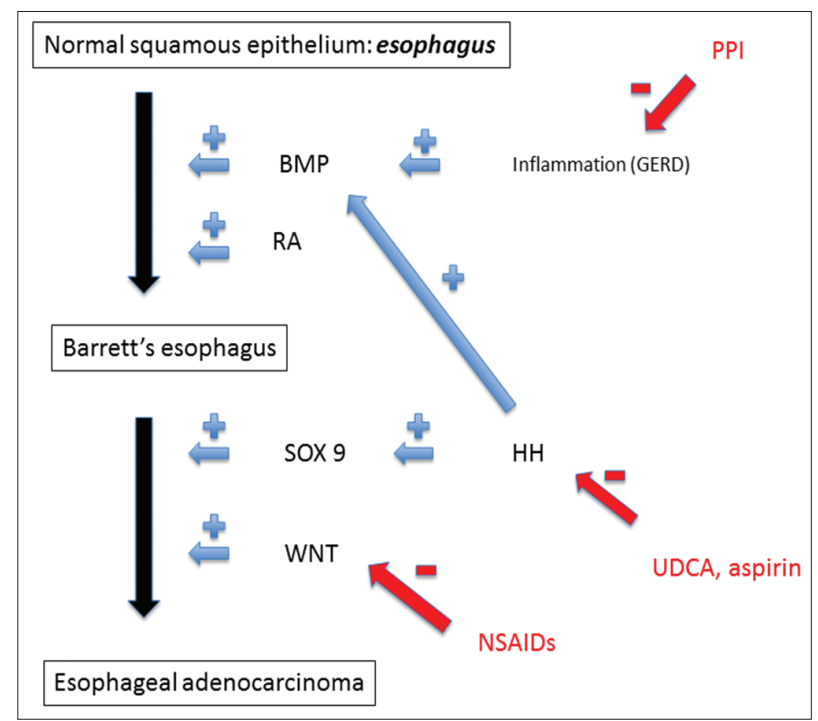

Figure 2 Molecular pathways leading to the progression of Barrett's esophagus to adenocarcinoma. Blue arrows indicate activation, red arrows therapeutic inhibition

$B M P$, bone morphogenetic protein; GERD, gastro-esophageal reflux disease; $R A$, retinoic acid signaling pathway; PPI, proton pump inhibitor; $H H$, hedgehog signaling pathway; WNT, winglesstype MMTV integration site family; UDCA, ursodeoxycholic acid; NSAIDs, non-steroid anti-inflammatory drugs

is indicated by the progressive increase in WNT signaling in the metaplasia-dysplasia-carcinoma sequence. In the development of $\mathrm{BE}$, activity of the RA signaling pathway is increased. In contrast, activation of this pathway reduces the development of EAC, which could be seen as a possible protective effect in progression.

Recently, two papers were published in Nature Genetics regarding the genetic changes involved in the progression from BE to EAC. In both studies, the authors used techniques such as whole-exome and whole-genome sequencing to provide more insight into the transition towards cancer. Ross-Innes, et al conducted whole-genome sequencing of paired EAC and BE samples with limited overlap between mutations [43]. The mutational signatures, however, were similar in the different lesions. This could be indicative of a comparable mutagenic process. Another whole-exome sequencing study found similar mutational signatures for EAC and BE [44]. Analysis of mutations in TP53 and cyclin-dependent kinase inhibitor 2A showed less prevalent oncogene activation events. They concluded that this probably occurred later in tumor progression. A model is presented whereby EAC can progress via two separate pathways: TP53 mutation followed by genome doubling versus progressive loss of tumor suppressors in those that do not undergo genome doubling.

\section{Chemoprevention}

To improve the survival of patients with EAC, the best strategy remains early diagnosis; however, the cancer often spreads before symptoms occur. BE is a known precursor to EAC, yet an ideal management strategy remains elusive and the utility of current endoscopic surveillance is controversial and costly. For these reasons, the use of chemoprevention strategies has gained considerable interest in recent studies, which aim to prevent the progression of BE towards EAC with pharmacological strategies.

Possible therapeutic targets according to the different molecular pathways involved in the progression of BE toward EAC can be summarized as downregulation of the Hh and WNT signaling pathways, and upregulation of the RA and (probably) the BMP signaling pathways. In the Hh signaling pathway, a possible target is the suppression of zinc-finger transcription factors GLI using a combination of ursodeoxycholic acid and aspirin. This significantly decreased the incidence of EAC in a rat model [45]. A second possibility is the use of WNT antagonists, such as non-steroid anti-inflammatory drugs (NSAIDs), to decrease the WNT signaling pathway and thereby reduce the risk of BE dysplasia and adenocarcinoma. The RA signaling pathway is a third potential therapeutic strategy; however, a clear target has not yet been described.

Several studies have reported a possible reduction in the risk of EAC among patients with BE with the use of NSAIDs, low-dose aspirin, statins and proton pump inhibitors (PPIs) [46-54]. These were all based on small, selected groups of patients with EAC. A meta-analysis, however, showed a significantly lower risk of esophageal cancer in patients who frequently used NSAIDs or aspirin [47]. This was confirmed for the risk of both EAC and HGD in another meta-analysis [55]. The effect of PPIs in the malignant transformation is still a matter for debate. PPIs are known as a treatment for symptom relief in BE, which suggests a decrease in the risk of progression. Nevertheless, certain studies show an increase in the risk of progression $[48,56]$. This could be explained by the use of PPIs in the treatment of GERD, a possible risk factor for EAC. According to recent studies, statins may also have potential for chemoprevention. In 2013, a systematic review and meta-analysis showed a reduction in the risk of EAC among patients with BE who were taking statins [57]. In 2014, Choi et al evaluated the effectiveness and cost-effectiveness of aspirin, statin, and combination chemoprevention for $\mathrm{BE}$ management [58]. They suggested that, among the four treatment strategies analyzed (endoscopic surveillance alone, aspirin chemoprevention, statin chemoprevention and the combination of aspirin and statin chemoprevention), aspirin therapy is the most cost-effective chemoprevention strategy for patients with BE. A combination of aspirin and statins could potentially be cost-effective in those patients with $\mathrm{BE}$ who have a higher risk of progression to EAC. A recent matched casecontrol study evaluated the risk of EAC among patients with $\mathrm{BE}$ associated with the use of NSAIDs, low-dose aspirin, statins and PPIs [59]. In this study, a non-significant reduction in the risk of HGD and EAC was only present when PPIs were used at the highest dose in patients with BE. The use of NSAIDs or lowdose aspirin was also not associated with a decrease in the risk of EAC. For statin use, a non-significant dose-duration response was seen. No statistically significant argument was found to indicate chemoprevention in daily practice for patients with $\mathrm{BE}$. 
To further investigate the possibility for chemoprevention, a large study centered in the United Kingdom, the Aspirin and Esomeprazole Chemoprevention in Barrett's Metaplasia (AspECT) study (http://www.octo-oxford.org.uk/alltrials/ infollowup/aspect.html), is evaluating the impact of low- and high-dose aspirin on BE progression rates to cancer. However, no similar trial that evaluates the impact of statins and the combination of a statin and aspirin is currently ongoing.

\section{Concluding remarks}

In view of the increasing incidence of EAC, it is important that more attention be paid to the prevention of this premalignant condition. However, the rate of progression towards adenocarcinoma is very small in comparison to what was thought previously. For this reason, it is important that risk factors for progression are identified to identify patients at risk.

Recently, a molecular revolution has occurred, with the identification of several possible factors contributing to the sequence towards adenocarcinoma. On this basis it may be possible to identify new targets for the treatment and prevention of adenocarcinoma.

In the future, a better understanding of the evolutionary dynamics of Barrett's clones is crucial for understanding the process of neoplastic progression. This will have important implications for the clinical management of the disease, as currently no good markers exist for the prediction of neoplastic progression. The ultimate goal should be to accurately identify on the one hand the low-risk Barrett cases and on the other hand the ones that are "born to be bad". Surveillance programs or chemoprevention can be applied more specifically to these groups of patients, but more research and improvement of current surveillance programs are still necessary.

\section{Acknowledgment}

Editorial support was provided by Mark English.

\section{References}

1. Spechler SJ. Barrett's esophagus. Semin Gastrointest Dis 1996;7:51-60.

2. Spechler SJ, Fitzgerald RC, Prasad GA, Wang KK. History, molecular mechanisms, and endoscopic treatment of Barrett's esophagus. Gastroenterology 2010;138:854-869.

3. Naini BV, Souza RF, Otze RD. Barrett's esophagus: a comprehensive and contemporary review for pathologists. Am J Surg Pathol 2016;40:e45-66.

4. Bennett C, Moayyedi P, Corley DA, et al; BOB CAT Consortium. BOB CAT: A large-scale review and Delphi consensus for management of Barrett's Esophagus with no dysplasia, indefinite for, or low-grade dysplasia. Am J Gastroenterol 2015;110:662-682.

5. Jankowski J, Barr H. Improving surveillance for Barrett's oesophagus: AspECT and BOSS trials provide an evidence base.
BMJ 2006;332:1512.

6. Rex DK, Cummings OW, Shaw M, et al. Screening for Barrett's esophagus in colonoscopy patients with and without heartburn. Gastroenterology 2003;125:1670-1677.

7. Ward EM, Wolfsen HC, Achem SR, et al. Barrett's esophagus is common in older men and women undergoing screening colonoscopy regardless of reflux symptoms. Am J Gastroenterol 2006;101:12-17.

8. Ronkainen J, Aro P, Storskrubb T, et al. Prevalence of Barrett's esophagus in the general population: an endoscopic study. Gastroenterology 2005;129:1825-1831.

9. Zagari RM, Fuccio L, Wallander MA, et al. Gastro-oesophageal reflux symptoms, oesophagitis and Barrett's oesophagus in the general population: the Loiano-Monghidoro study. Gut 2008;57:1354-1359.

10. Zou D, He J, Ma X, et al. Epidemiology of symptom-defined gastroesophageal reflux disease and reflux esophagitis: the systematic investigation of gastrointestinal diseases in China (SILC). Scand J Gastroenterol 2011;46:133-141.

11. Rubenstein JH, Inadomi JM, Scheiman J, et al. Association between Helicobacter pylori and Barrett's esophagus, erosive esophagitis, and gastroesophageal reflux symptoms. Clin Gastroenterol Hepatol 2014; 12:239-245.

12. de Jonge PJ, van Blankenstein M, Grady WM, Kuipers EJ. Barrett's oesophagus: epidemiology, cancer risk and implications for management. Gut 2014;63:191-202.

13. Cook MB, Chow WH, Devesa SS. Oesophageal cancer incidence in the United States by race, sex, and histologic type, 1977-2005. Br J Cancer 2009;101:855-859.

14. Alvarez Herrero L, Curvers WL, van Vilsteren FG, et al. Validation of the Prague C\&M classification of Barrett's esophagus in clinical practice. Endoscopy 2013;45:876-882.

15. Salemme M, Villanacci V, Cengia G, Cestari R, Missale G, Bassotti G. Intestinal metaplasia in Barrett's oesophagus: An essential factor to predict the risk of dysplasia and cancer development. Dig Liver Dis 2016;48:144-147.

16. Shaheen NJ, Crosby MA, Bozymski EM, Sandler RS. Is there publication bias in the reporting of cancer risk in Barrett's esophagus? Gastroenterology 2000;119:333-338.

17. Chang EY, Morris CD, Seltman AK, et al. The effect of antireflux surgery on esophageal carcinogenesis in patients with Barrett esophagus: a systematic review. Ann Surg 2007;246:11-21.

18. Thomas T, Abrams KR, De Caestecker JS, Robinson RJ. Meta analysis: Cancer risk in Barrett's oesophagus. Aliment Pharmacol Ther 2007;26:1465-1477.

19. Yousef F, Cardwell C, Cantwell MM, Galway K, Johnston BT, Murray L. The incidence of esophageal cancer and high-grade dysplasia in Barrett's esophagus: a systematic review and metaanalysis. Am J Epidemiol 2008;168:237-249.

20. Sikkema M, de Jonge PJ, Steyerberg EW, Kuipers EJ. Risk of esophageal adenocarcinoma and mortality in patients with Barrett's esophagus: a systematic review and meta-analysis. Clin Gastroenterol Hepatol 2010;8:235-244.

21. Wani S, Puli SR, Shaheen NJ, et al. Esophageal adenocarcinoma in Barrett's esophagus after endoscopic ablative therapy: a meta-analysis and systematic review. Am $J$ Gastroenterol 2009;104:502-513.

22. Desai TK, Krishnan K, Samala N, et al. The incidence of oesophageal adenocarcinoma in non-dysplastic Barrett's oesophagus: a metaanalysis. Gut 2012;61:970-976.

23. de Jonge PJ, van Blankenstein M, Looman CW, Casparie MK, Meijer GA, Kuipers EJ. Risk of malignant progression in patients with Barrett's oesophagus: a Dutch nationwide cohort study. Gut 2010;59:1030-1036.

24. Hvid-Jensen F, Pedersen L, Drewes AM, Sørensen HT, Funch-Jensen P. Incidence of adenocarcinoma among patients 
with Barrett's esophagus. N Engl J Med 2011;365:1375-1383.

25. van der Burgh A, Dees J, Hop WC, van Blankenstein M. Oesophageal cancer is an uncommon cause of death in patients with Barrett's oesophagus. Gut 1996;39:5-8.

26. Macdonald CE, Wicks AC, Playford RJ. Final results from 10 year cohort of patients undergoing surveillance for Barrett's oesophagus: observational study. BMJ 2000;321:1252-1255.

27. Dulai GS, Guha S, Kahn KL, Gornbein J, Weinstein WM. Preoperative prevalence of Barrett's esophagus in esophageal adenocarcinoma: a systematic review. Gastroenterology 2002;122:26-33.

28. Curvers WL, Peters FP, Elzer B, et al. Quality of Barrett's surveillance in The Netherlands: a standardized review of endoscopy and pathology reports. Eur J Gastroenterol Hepatol 2008;20:601-607.

29. Sharma P, Hawes RH, Bansal A, et al. Standard endoscopy with random biopsies versus narrow band imaging targeted biopsies in Barrett's oesophagus: a prospective, international, randomised controlled trial. Gut 2013;62:15-21.

30. Fitzgerald RC, di Pietro M, Ragunath K, et al; British Society of Gastroenterology. British Society of Gastroenterology guidelines on the diagnosis and management of Barrett's oesophagus. Gut 2014;63:7-42.

31. Kerkhof M, van Dekken H, Steyerberg EW, et al; CYBAR study group. Grading of dysplasia in Barrett's oesophagus: substantial interobserver variation between general and gastrointestinal pathologists. Histopathology 2007;50:920-927.

32. Ross-Innes CS, Debiram-Beecham I, O'Donovan M, et al. Evaluation of a minimally invasive cell sampling device coupled with assessment of trefoil factor 3 expression for diagnosing Barrett's esophagus: a multi-center case-control study. PLoS Med 2015;12:e1001780.

33. Benaglia T, Sharples LD, Fitzgerald RC, Lyratzopoulos G. Health benefits and cost effectiveness of endoscopic and nonendoscopic cytosponge screening for Barrett's esophagus. Gastroenterology 2013;144:62-73.

34. Chevaux JB, Piessevaux H, Jouret-Mourin A, Yeung R, Danse E, Deprez PH. Clinical outcome in patients treated with endoscopic submucosal dissection for superficial Barrett's neoplasia. Endoscopy 2015;47:103-112.

35. Prasad GA, Bansal A, Sharma P, Wang KK. Predictors of progression in Barrett's esophagus: current knowledge and future directions. Am J Gastroenterol 2010;105:1490-1502.

36. Verbeek RE, Spittuler LF, Peute A, et al. Familial clustering of Barrett's esophagus and esophageal adenocarcinoma in a European cohort. Clin Gastroenterol Hepatol 2014;12:1656-1663.e1.

37. Singh S, Sharma AN, Murad MH, et al. Central adiposity is associated with increased risk of esophageal inflammation, metaplasia, and adenocarcinoma: a systematic review and metaanalysis. Clin Gastroenterol Hepatol 2013;11:1399-1412.

38. Thrift AP, Garcia JM, El-Serag HB. A multibiomarker risk score helps predict risk for Barrett's esophagus. Clin Gastroenterol Hepatol 2014;12:1267-1271.

39. Duggan C, Onstad L, Hardikar S, Blount PL, Reid BJ, Vaughan TL. Association between markers of obesity and progression from Barrett's esophagus to esophageal adenocarcinoma. Clin Gastroenterol Hepatol 2013;11:934-943.

40. Chandrasoma PT, Der R, Dalton P, et al. Distribution and significance of epithelial types in columnar-lined esophagus. Am J Surg Pathol 2001;25:1188-1193.

41. McDonald SAC, Lavery D, Wright NA, Jansen M. Barrett esophagus: lessons on its origins from the lesion itself. Nat Rev Gastroenterol Hepatol 2015;12:50-60.

42. Pavlov K, Meijer C, van den Berg A, Peters FT, Kruyt FA, Kleibeuker JH. Embryological signaling pathways in Barrett's metaplasia development and malignant transformation; mechanisms and therapeutic opportunities. Crit Rev Oncol
Hematol 2014;92:25-37.

43. Ross-Innes CS, Becq J, Warren A, et al; Oesophageal Cancer Clinical and Molecular Stratification OCCAMS Study Group. Whole-genome sequencing provides new insights into the clonal architecture of Barrett's esophagus and esophageal adenocarcinoma. Nat Genet 2015;47:1038-1046.

44. Stachler MD, Taylor-Weiner A, Peng S, et al. Paired exome analysis of Barrett's esophagus and adenocarcinoma. Nat Genet 2015;47:1047-1055.

45. Rizvi S, Demars CJ, Comba A, et al. Combinatorial chemoprevention reveals a novel smoothened-independent role of GLI1 in esophageal carcinogenesis. Cancer Res 2010;70:6787-6796.

46. Beales IL, Vardi I, Dearman L. Regular statin and aspirin use in patients with Barrett's oesophagus is associated with a reduced incidence of oesophageal adenocarcinoma. Eur J Gastroenterol Hepatol 2012;24:917-923.

47. Corley DA, Kerlikowske K, Verma R, Buffler P. Protective association of aspirin/NSAIDs and esophageal cancer: a systematic review and meta-analysis. Gastroenterology 2003;124:47-56.

48. Nguyen DM, Richardson P, El-Serag HB. Medications (NSAIDs, statins, proton pump inhibitors) and the risk of esophageal adenocarcinoma in patients with Barrett's esophagus. Gastroenterology 2010;138:2260-2266.

49. Kantor ED, Onstad L, Blount PL, Reid BJ, Vaughan TL. Use of statin medications and risk of esophageal adenocarcinoma in persons with Barrett's esophagus. Cancer Epidemiol Biomarkers Prev 2012;21:456-461.

50. Kastelein F, Spaander MC, Biermann K, Steyerberg EW, Kuipers EJ, Bruno M); Probar-study Group. Nonsteroidal anti-inflammatory drugs and statins have chemopreventative effects in patients with Barrett's esophagus. Gastroenterology 2011;141:2000-2008; quiz e13-e14.

51. Abnet CC, Freedman ND, Kamangar F, Leitzmann MF, Hollenbeck AR, Schatzkin A. Non-steroidal anti-inflammatory drugs and risk of gastric and oesophageal adenocarcinomas: results from a cohort study and a meta-analysis. Br J Cancer 2009;100:551-557.

52. Rothwell PM, Fowkes FG, Belch JF, Ogawa H, Warlow CP, Meade TW. Effect of daily aspirin on long-term risk of death due to cancer: analysis of individual patient data from randomised trials. Lancet 2011;377:31-41.

53. Singh S, Garg SK, Singh PP, Iyer PG, El-Serag HB. Acid-suppressive medications and risk of oesophageal adenocarcinoma in patients with Barrett's oesophagus: a systematic review and meta-analysis. Gut 2014;63:1229-1237.

54. Sivarasan N, Smith G. Role of aspirin in chemoprevention of esophageal adenocarcinoma: a meta-analysis. J Dig Dis 2013;14:222-230.

55. Zhang S, Zhang XQ, Ding XW, et al. Cyclooxygenase inhibitors use is associated with reduced risk of esophageal adenocarcinoma in patients with Barrett's esophagus: a meta-analysis. $\mathrm{Br} J$ Cancer 2014;110:2378-2388.

56. García Rodríguez LA, Lagergren J, Lindblad M. Gastric acid suppression and risk of oesophageal and gastric adenocarcinoma: a nested case control study in the UK. Gut 2006;55:1538-1544.

57. Singh S, Singh AG, Singh PP, Murad MH, Iyer PG. Statins are associated with reduced risk of esophageal cancer, particularly in patients with Barrett's esophagus: a systematic review and metaanalysis. Clin Gastroenterol Hepatol 2013;11:620-629.

58. Choi SE, Perzan KE, Tramontano AC, Kong CY, Hur C. Statins and aspirin for chemoprevention in Barrett's oesophagus: results of a cost-effectiveness analysis. Cancer Prev Res (Phila) 2014;7:341-350.

59. Masclee GM, Coloma PM, Spaander MC, Kuipers EJ, Sturkenboom MC. NSAIDs, statins, low-dose aspirin and PPIs, and the risk of oesophageal adenocarcinoma among patients with Barrett's oesophagus: a population-based case-control study. BMJ Open 2015;5:e006640. 